\title{
Coupled Resonances in Multiple Silicon Photonic Crystal Cavities in All-Optical Solid-State Analogy to Electromagnetically Induced Transparency
}

Xiaodong Yang, Member, IEEE, Mingbin Yu, Dim-Lee Kwong, Fellow, IEEE, and Chee Wei Wong, Member, IEEE

(Invited Paper)

\begin{abstract}
We present experimental and theoretical studies of coupled resonances in multiple silicon photonic crystal cavities in an all-optical classical analogy to electromagnetically induced transparency. Multiple implementation schemes are demonstrated, such as two (in various configurations), and four cavities. The photonic crystal cavities are embedded in 2-D photonic crystal slabs and designed in the overcoupled regime. Our observations include measured transparency resonance lifetimes more than three times the single-cavity lifetimes, Fano-like lineshapes, and stepwise resonance control through multiple aligned external optical pump beams. All observed schemes are analyzed rigorously through finite-difference time-domain simulations and the coupled-mode formalism. Our experimental and theoretical results are applicable to optical pulse trapping and all-optical dynamical storage of light in the solid state.
\end{abstract}

Index Terms-Electromagnetically induced transparency, optical cavities, photonic crystals, slow light.

\section{INTRODUCTION}

$\mathbf{P}$ HOTONIC crystal nanocavities in 2-D photonic crystal slabs with remarkably high quality factors $(Q)$ and wavelength-scale modal volumes $\left(V_{\mathrm{m}}\right)$ have been achieved recently [1], [2]. The strong photon localization and long photon lifetimes in these high- $Q / V_{\mathrm{m}}$ photonic crystal nanocavities point to enhanced light-matter interactions, such as thermooptic nonlinearity and bistability [3], [4], Raman lasing [5], [6], digital resonance tuning [7], and cavity quantum electrodynamics [8] in silicon nanophotonics. In-plane-type devices in 2-D photonic crystal slabs have been realized with high- $Q / V_{\mathrm{m}}$ photonic crystal nanocavities, such as channel drop filters [9], Fano interference [4], and dynamic control of the $Q$ factor of nanocavities [10].

Manuscript received April 29, 2009; revised August 24, 2009. Current version published February 5, 2010. This work was supported in part by the Defense Advanced Research Projects Agency (DARPA) Young Faculty Award, by the National Science Foundation (NSF) CAREER award (ECCS 0747787), and by the New York State Office of Science, Technology and Academic Research. The work of X. Yang was supported by an Intel Fellowship.

X. Yang and C. W. Wong are with the Optical Nanostructures Laboratory, Columbia University, New York, NY 10028 USA (e-mail: xy2103@columbia. edu; cww2104@columbia.edu).

M. Yu and D.-L. Kwong are with the Institute of Microelectronics, Singapore 117685, Singapore (e-mail: mingbin@ime.a-star.edu.sg; kwongdl@ ime.a-star.edu.sg).

Color versions of one or more of the figures in this paper are available online at http://ieeexplore.ieee.org.

Digital Object Identifier 10.1109/JSTQE.2009.2032665
The all-optical analog to electromagnetically induced transparency (EIT) [11] in 2-D photonic crystal cavities with dynamical tuning has been proposed toward trapping photons, time reversal, and even ultimately stopping light beyond the previously conceived delay-bandwidth limit [12]-[14]. Using a photonic crystal waveguide side-coupled to cavities system, this phenomenon has been rigorously examined theoretically [15], [16]. Recently, theoretical analyses in coupled whispering-gallerymode resonators have revealed the analogy between the coherent effects in the classical system and EIT in an atomic system [17], [18]. EIT-like effects were examined experimentally in coupled whispering-gallery mode resonators, such as ultrahigh- $Q$ silica microspheres [19], [20] and microring resonators [21]-[24], with observations of slow group velocities and storing light onchip beyond the static bandwidth limit.

In EIT, the quantum destructive interference between transitions to a common energy level in atoms has led to a sharp cancellation of absorption in the medium [11], resulting in phenomena such as freezing light [25] and dynamical storage of light in a solid-state system [26]. Fig. 1(a) illustrates the physics of EIT in a three-level lambda-type scheme, which involves two distinct polarization-sensitive resonances, $\omega_{p}$ and $\omega_{c}$. The atomic transitions from $|1\rangle$ to $|3\rangle$ and $|2\rangle$ to $|3\rangle$ define the absorption of the probe laser field with frequency $\omega_{p}$ and the control laser field with frequency $\omega_{c}$, respectively. The destructive interference between the two absorption amplitudes, including different excitation pathways from $|1\rangle-|3\rangle$ directly and from $|1\rangle-|3\rangle-|2\rangle-|3\rangle$ indirectly, leads to an EIT transparent window between two dressed states $|3 d\rangle$ and $|2 d\rangle$. Similar to atomic systems, where the interference is driven by a coherent laser field, EIT-like effects can be remarkably observed through classical means. For optical analog to EIT, as described in Fig. 1(b), each optical resonance with frequency of $\omega_{A}$ and $\omega_{B}$ from two optical resonators is analog to the transitions between the energy levels in the atomic EIT system. The coupling strength $\Omega_{c}$ between the two resonators acts as the control laser field, which is determined by the frequency detuning $\left(\omega_{A}-\omega_{B}\right)$. The coherent interference between two optical pathways will introduce EIT transparency region between two resonances $\omega_{A}$ and $\omega_{B}$.

Here, we present detailed experimental observations of alloptical analog to EIT in coherently coupled standing wave photonic crystal cavities, with deterministic resonance- and phase tuning through thermo-optic tuning method. This furthers from our initial results shown in [27], with zero-separation $(L=0)$ 
(a)

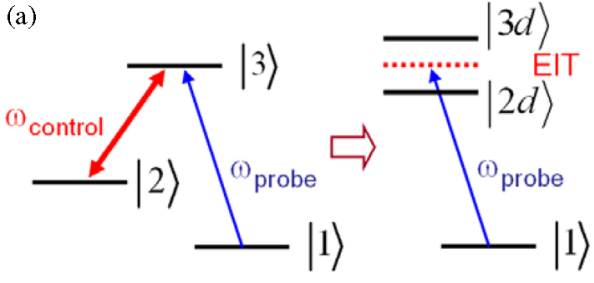

(b)

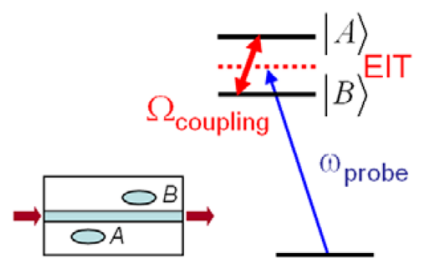

Fig. 1. (a) In three-level Lambda-type scheme, the destructive interference between the probe absorption amplitudes leads to EIT. The laser fields can drive population from $|1\rangle-|3\rangle$ directly or from $|1\rangle-|3\rangle-|2\rangle-|3\rangle$ indirectly. These two probability amplitudes for the different excitation pathways interfere destructively to create an EIT transparent window between two dressed states $|3 \mathrm{~d}\rangle$ and $|2 d\rangle$. (b) In optical analog to EIT, each optical resonance with frequency of $\omega_{A}$ and $\omega_{B}$ from two optical resonators is analog to the transitions between the energy levels in the atomic EIT system. The coupling strength $\Omega_{c}$ between the two resonators acts as the control laser field. The coherent interference between two optical pathways will introduce an EIT-like transparency region between two resonances $\omega_{A}$ and $\omega_{B}$.

between cavities, four cavity designs and initial measurements, and detailed numerical simulations to support our measurements. Our results demonstrate EIT-like transparency resonance lifetimes more than three times the single-cavity lifetime, cavity resonance tuning, and a full-range control in phase ( 0 to $\pi$ ). The coupled photonic crystal cavities through a single "bus" waveguide for coherent light manipulation overcome much of the limitations on decoherence and bandwidth in atomic systems, allowing applications toward on-chip optical pulse trapping and all-optical dynamical storage of light.

\section{DESIGN OF OPTICAL EIT-LIKE SYSTEM}

The optical EIT-like system (Fig. 2) consists of a photonic crystal waveguide side-coupled to two, three, or more high intrinsic quality factor $(Q)$ photonic crystal cavities. Each cavity consists of three missing air holes (L3) in an air-bridge triangular lattice photonic crystal slab with thickness of $0.6 a$ and the radius of air holes is $0.29 a$, where the lattice period $a=$ $420 \mathrm{~nm}$, such as examined in [12] and [28]. Each cavity has the nearest neighbor holes at the cavity edge that are shifted $\left(S_{1}\right)$ by $0.15 a$ to tune the radiation mode field for increased intrinsic $Q$ factors [29]. In order to optimize the in-plane optical couplings between the $L 3$ cavity and the photonic crystal waveguide, the coupling quality factor $Q_{c}$, the intrinsic quality factor $Q_{\text {int }}$, and the total quality factor $Q_{\text {tot }}$ as a function of the number of lattice layers in-between is developed with full 3D finite-difference time-domain (FDTD) numerical simulations (MEEP software developed in MIT) [30]. $Q$ is directly calculated from the total energy in the cavity and the power outputted from the cavity [31]. The calculated modal volume is (a)

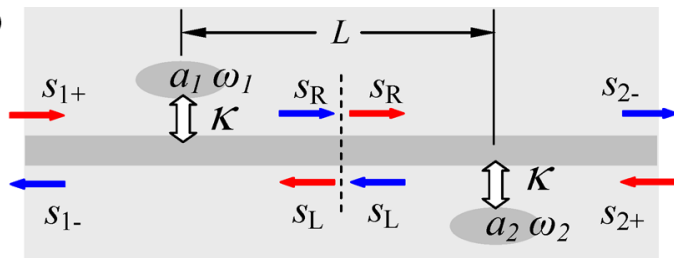

(b)

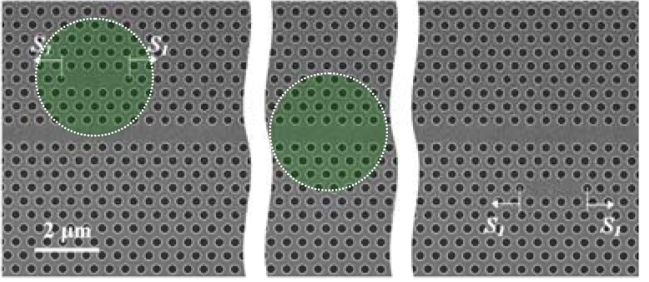

(c)

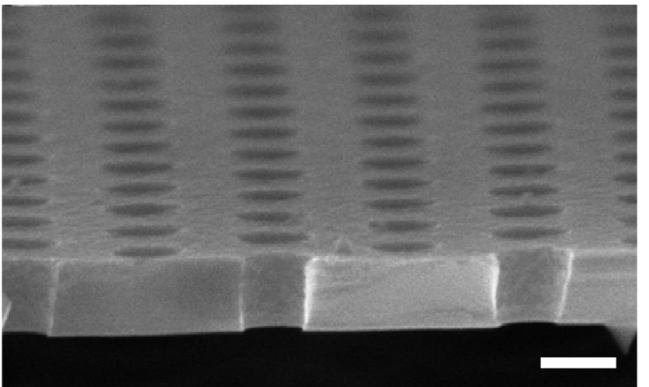

Fig. 2. (a) Schematic of optical EIT-like system including a waveguide sidecoupled to two or more cavities. (b) Example SEM of two photonic crystal L3 cavities side-coupled to a single photonic crystal waveguide. Each cavity has the nearest neighbour holes at the cavity edge tuned by $S_{1}=0.15 a$ to suppress the vertical radiation loss by more than an order of magnitude compared to the coupling rate. The green spots indicate two focused pump laser beams used for thermo-optic tuning. (c) Cross-section SEM to illustrate suspended membrane samples on all our measurements. Scale bar: $200 \mathrm{~nm}$. (a)

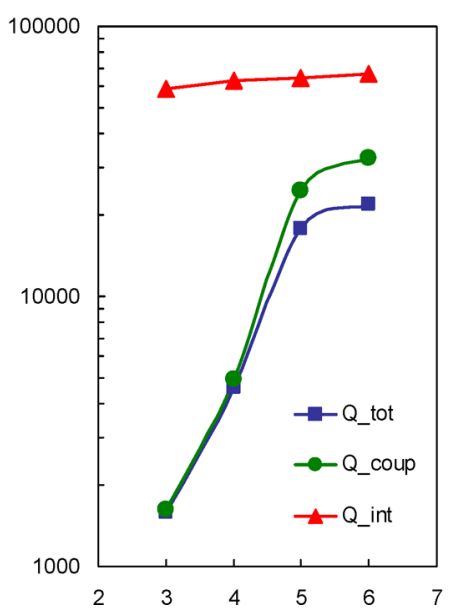

(b)

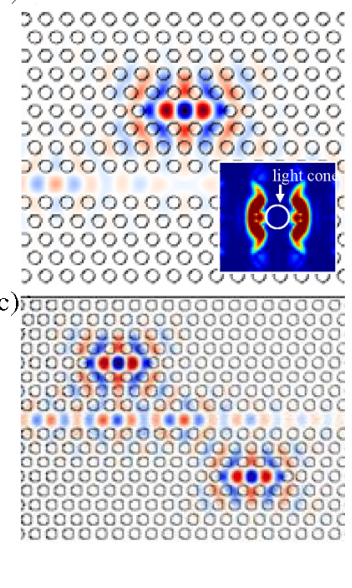

Fig. 3. (a) The $Q_{\text {int }}, Q_{v}$, and $Q_{\text {tot }}$ as a function of the number of lattice layers between the $L 3$ cavity and the photonic crystal waveguide calculated with 3-D FDTD method. (b) $E_{y}$ field of the fundamental mode of single $L 3$ cavity for waveguide-to-cavity separation of three lattice layers. Inset: $k$-space amplitudes for single $L 3$ cavity, illustrating high radiation $Q$ factor. (c) $E_{y}$-field intensity of the coupled two-cavity transparency mode at midslab.

$\sim 0.74$ cubic wavelengths $\left[(\lambda / n)^{3}\right]$. As shown in Fig. 3(a), for a single tuned $\left(s_{1}=0.15 a\right) L 3$ cavity coupled to the photonic crystal waveguide, $Q_{c}$ has a strong dependence on the number of lattice layers between the cavity and the waveguide, while $Q_{\text {int }}$ 
is almost constant $\sim 60,000$ for all the different lattice layers. For three lattice layers (the center-to-center waveguide-to-cavity separation is $2 \sqrt{3} a$ ), $Q_{c}$ is around 1600 , and $Q_{\mathrm{tot}} \sim 1560$. In this paper, we deliberately designed the ratio of $Q_{\text {int }}$ to $Q_{c}$ to be high ( 37.5) in each cavity, so as to operate in the overcoupled regime with vertical radiation loss well suppressed for in-plane cavity-cavity interference. With tuning of three air holes at cavity edge, the $Q_{\text {int }}$ can reach up to 100,000 , which will reduce the vertical radiation loss further more [32]. Fig. 3(b) shows the $E_{y}$ field of the resonance mode midslab from 3-D FDTD simulation for waveguide-to-cavity separation of three lattice layers.

In our five example device implementations shown in this paper, the in-plane center-to-center separation $L$ between the cavities ranges from zero (sample 1), $10 a$ (sample 2), 11 $a$ (sample 3), $12 a$ (sample 5), to $142 a$ (sample 4). When the resonance frequencies of two cavities $\omega_{1}$ and $\omega_{2}$ overlap (with $\delta=2 \tau_{\text {total }}\left(\omega_{1}-\omega_{2}\right) \neq 0$, where $\tau_{\text {total }}$ is the loaded lifetime of individual cavity) and the round-trip phase $2 \phi$ between two cavities satisfy the condition of forming a Fabry-Pérot resonance $(2 n \pi)$, the current system represents an all-optical analog of atomic EIT. The system will support two degenerate modes at the frequency of $\left(\omega_{1}+\omega_{2}\right) / 2$. One mode decays slower compared to a single cavity, the other mode decays faster. The mode with decay slower than the single-cavity lifetime gives rise to the EIT-like spectral feature [33]. When $\delta<\sim 3.5, Q_{\mathrm{EIT}}>Q_{\text {total }}$ so that the condition of EIT-like coherent interference is satisfied. Fig. 3(c) shows the $E_{y}$-field intensities of transparency mode in the dark state of the two coupled $L 3$ cavities from 3-D FDTD simulation.

\section{EXPERIMENTAL RESULTS}

The devices are fabricated with 248-nm deep UV lithography in a silicon-on-insulator substrate. The silicon layer is $250 \mathrm{~nm}$ thick and on top of $1 \mu \mathrm{m}$ buried oxide, which is sacrificially etched to form air-bridged photonic crystal slabs. An example SEM of one of the device samples is shown in Fig. 2(b). The measured lattice period is bounded within $422.97 \pm 1.65 \mathrm{~nm}$ with hole radius of $121.34 \pm 1.56 \mathrm{~nm}$, and a feature ellipticity of $1.57 \pm 0.79 \mathrm{~nm}$ [34]. An in-line fiber polarizer and a polarization controller are used to couple TE polarization light from an amplified spontaneous emission source ranging from 1525 to $1610 \mathrm{~nm}$ into the waveguide through a tapered lensed fiber. A second tapered lensed fiber collects the transmission from the waveguide output that is sent to an optical spectrum analyzer with 10 -pm resolution.

For sample 1, the distance $L$ between two cavities is zero, as shown in Fig. 4, which is a particular case to satisfy the condition of forming a Fabry-Pérot resonance (the round-trip phase between the two cavities is zero) [14]. The current system is in consistency with the case of nonorthogonal modes in R[15], which represents an all-optical analog of atomic EIT. Fig. 5(a) shows the measured transmission spectrum of the EIT-like system with $L=0$. The EIT-like lineshape is apparent and the fullwidth at half maximum (FWHM) of the EIT-like transparency peak is $\sim 0.2 \mathrm{~nm}$, corresponding to a $Q_{\text {EIT }}$ of 7500 , which is much higher than the measured $Q_{\text {tot }}$ of each $L 3$ nanocavity,
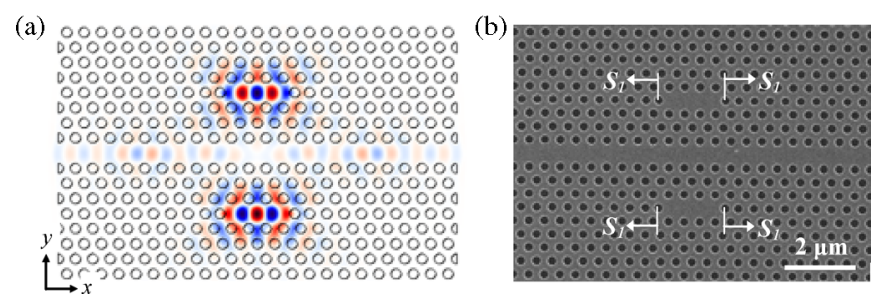

Fig. 4. (a) $E_{y}$-field intensity of the coupled-cavity transparency mode at midslab for $L=0$. (b) SEM images of two photonic crystal $L 3$ cavities sidecoupled to a photonic crystal waveguide for $L=0$. Each cavity has the nearest neighbor holes at the cavity edge tuned by $s_{1}=0.15 a$ to suppress the vertical radiation loss by more than an order of magnitude compared to the coupling rate.
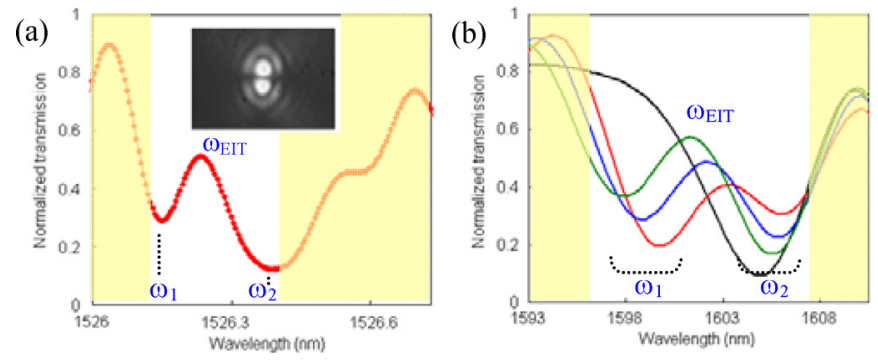

Fig. 5. (a) Measured transmission lineshape of the EIT-like system for sample $1(L=0)$. Inset: Near-field image of the light emitted from the defect region on the transparency peak $(1526.21 \mathrm{~nm})$. (b) The EIT-like transmission spectra with different wavelength detuning calculated from 3-D FDTD method. The black line denotes the single cavity resonance.

which is $\sim 1900$. The width of the EIT-like peak, indicating the coherent interference between the two cavity modes, is dependent on the wavelength detuning $\left(\omega_{1}-\omega_{2}\right)$, which is induced by the fabrication nonperfection in the present sample and is not tunable. The IR image at the wavelength of EIT transparency peak is shown in the inset of Fig. 5(a), showing the strong optical excitation of both nanocavities for coherent interference between them. Fig. 4(a) shows the $E_{y}$ field distribution of the transparency resonance mode with 3-D FDTD method. The dielectric constant is 12.11 and 11.975 for the upper cavity and the lower cavity, respectively. The dielectric constant of the silicon slab is 12.11. Fig. 5(b) plots the calculated EIT-like transmission spectra with different dielectric constants of 11.975 (longest resonant wavelength), 11.95 (middle resonance), and 11.925 (shortest resonant wavelength) for the lower cavity. The quality factor of EIT transparency peak is around doubled compared to single $L 3$ cavity (black line). As the dielectric constants of two nanocavities are closer to each other, so as to the two resonant frequencies, the width of the transparency peak gets narrower.

The transmission lineshape of the optical EIT system is dependent on the resonance detuning $\delta$ between two cavities and the cavity-cavity round-trip phase $2 \phi$. Next, we use a thermooptic method to tune both $\delta$ and $\phi$ simultaneously. Two $532 \mathrm{~nm}$ continuous-wave green lasers are focused to within a $5 \mu \mathrm{m}$ spot size at both a cavity region and the connecting waveguide region [Fig. 6(a)] through an objective lens to locally perturb the silicon refractive index [35]. For silicon, the temperature dependence of the refractive index $d n / d T$ is $1.85 \times 10^{-4} / \mathrm{K}$ at room 
(a)

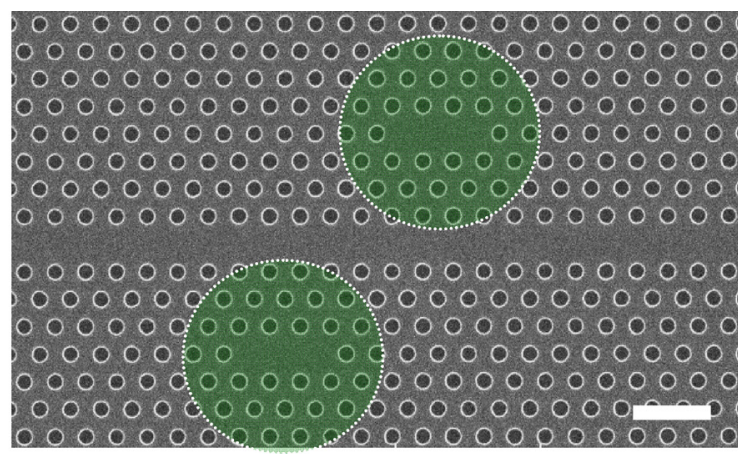

(b)

(i)

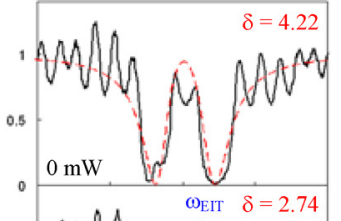

(ii)
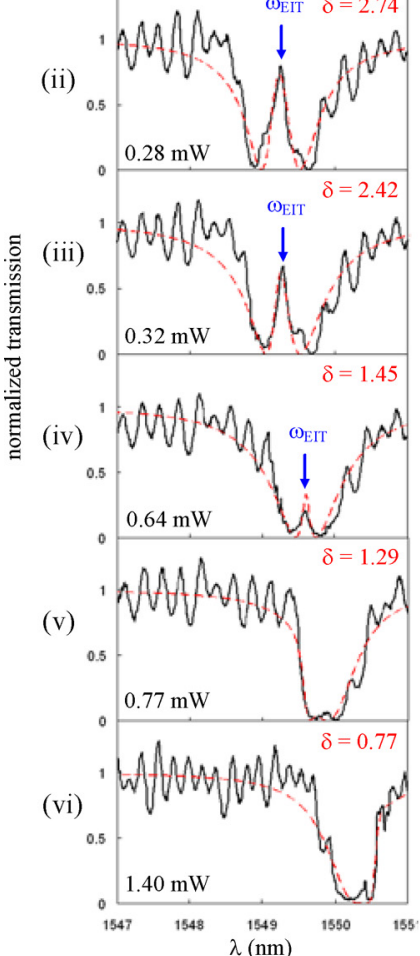

(c)

(i)

$$
\delta=4.45
$$

(ii)

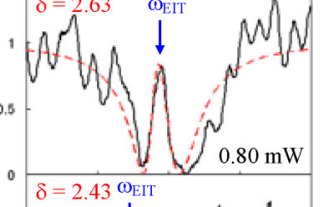

(iii)

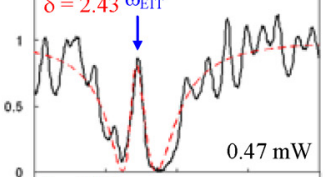

(iv)
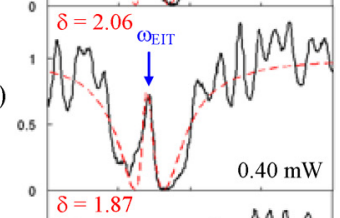

(v)

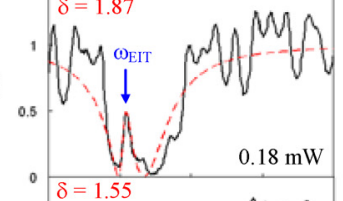

(vi)

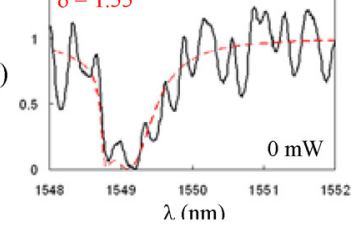

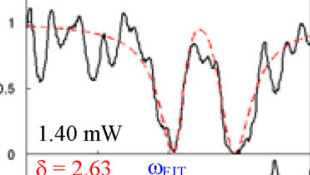

Fig. 6. (a) SEM image of two photonic crystal $L 3$ cavities side-coupled to a photonic crystal waveguide, where the green circles illustrate two focused pump laser beams used for thermo-optic tuning. (b) Measured and theoretical transmission lineshapes with various detuning $\left(\delta=2 \tau_{\text {total }}\left(\omega_{1}-\omega_{2}\right)\right.$, where $\tau_{\text {total }}$ is the loaded lifetime of individual cavity) for sample $2(L=11 a)$. (c) Measured and theoretical transmission lineshapes with various detuning for sample $3(L=10 a)$. Solid black lines show experimental data and red dashed lines show theoretical fits.

temperature. Pump positions and powers are carefully selected so that both $\delta$ and $\phi$ are precisely controlled for coherent interference.

The solid curves in Fig. 6(b) illustrate the measured transmission spectra of the EIT-like photonic crystal system for various pump powers (from 0 to $1.40 \mathrm{~mW}$ ) for sample 2. The linear relationship between the wavelength shift and the pump power is $\sim 1.32 \mathrm{~nm} / \mathrm{mW}$. Fig. 6(b)(i) shows the initial transmission spectrum for two uncoupled standing wave cavities, with two separated Lorentzian lineshapes, where the cavity resonances are $\lambda_{1}=1548.63$ and $\lambda_{2}=1549.45 \mathrm{~nm}$, respectively. The fabrication nonperfection results in the difference of two cavity resonances, which is $0.82 \mathrm{~nm}$ for sample 2. Measured total (loaded) quality factors for single cavities are $Q_{\text {tot,1 }} \sim 4000$ and $Q_{\text {tot } 2} \sim 3600$, estimated from the full-width at half-maximum (FWHM), and the intrinsic cavity $Q_{\text {int }}$ is $\sim 60,000$, as estimated from the $\sim 22.5 \mathrm{~dB}$ transmission dips. The discrepancy between measured and calculated total quality factors is due to the fluctuation of the resonance frequency of the cavity mode [9]. As the pump power is increased, both cavity resonances are red-shifted and the resonance detuning between two cavities $(\delta \omega)$ is narrower. Cavity 1 is located closer to the pump laser spot and has larger wavelength red shift than cavity 2 . For example, when the pump power is $0.28 \mathrm{~mW}$, the detuning $\delta$ is 2.74, and an EIT-like transparency peak is distinctively observed. With further decrease in detuning $\delta$, the transparency peak gets progressively narrower, shown in Fig. 6(a)(ii)-(iv), indicating increased coherent interference between two cavities. The FWHM of the symmetric transparency peak in Fig. 6(a)(iv) is $\sim 0.12 \mathrm{~nm}$, or a $Q_{\text {EIT }}$ of 13,000 , sizably larger than the loaded $Q$ of each L3 cavity and a longer photon delay than two noninteracting cavity lifetimes. Moreover, under stronger pump excitation, Fig. 6(a)(v) and (vi) now shows asymmetric Fano-like lineshapes ranging from the higher frequency edge [Fig. 6(a)(v)] to the lower frequency edge [Fig. 6(a)(vi)], due to departure of the round-trip phase $(2 \phi)$ from $2 n \pi$. In these figures, we show the unprocessed measurement data, with Fabry-Pérot noise oscillations at the side frequencies due to the reflection at the waveguide facets, which can be removed with integrated fiber-to-strip waveguide spot-size converter [36]. Furthermore, we verified the mode field distributions under controlled tuning, where for the example case of Fig. 6(a)(iii), radiation from both cavities is observed when the input laser is tuned to $1549.29 \mathrm{~nm}$. When detuned from the transparency peak and at either $1549.08 \mathrm{~nm}$ or $1549.55 \mathrm{~nm}$, only a single cavity predominantly radiates with the even symmetry of fundamental cavity mode. These EIT-like observations are further confirmed with sample 3, as shown in Fig. 6(c), where two cavities have very small resonance detuning initially, and the resonance wavelengths are $\lambda_{1}=1549.10 \mathrm{~nm}$ and $\lambda_{2}=1548.80 \mathrm{~nm}$, respectively. The measured total quality factors are $Q_{\text {tot, } 1} \sim 4100$ and $Q_{\text {tot }, 2} \sim 3100$. Without any external pumping, the transmission spectrum shows asymmetric Fano-like lineshapes [in Fig. 6(c)(vi)]. With pump tuning, the two cavity resonances separate and the strong in-plane coherent coupling results in clear observations of the EIT-like lineshapes, as shown in Fig. 6(c)(ii)-(v). The FWHM of the transparency peak in Fig. 6(c)(v) is $\sim 0.15 \mathrm{~nm}$, or a $Q_{\mathrm{EIT}}$ of 10,400 .

In the previous two series of experiments, the pump light influences both the cavity-cavity detuning $\delta$ and phase difference $\phi$ due to the limited separation between two cavities. In order to control $\delta$ and $\phi$ independently with two pump laser beams, the in-plane separation of two cavities is designed to be $L=142 a$ (or $\cong 60 \mu \mathrm{m}$ ). Fig. 2(b) shows the SEM picture of the fabricated device. The initial resonance wavelengths are $\lambda_{1}=1535.80 \mathrm{~nm}$ and $\lambda_{2}=1539.01 \mathrm{~nm}$, respectively. The measured total quality factors $Q_{\text {tot,1 }}$ and $Q_{\text {tot }, 2}$ are $~ 4000$. As illustrated in Fig. 2(b), 

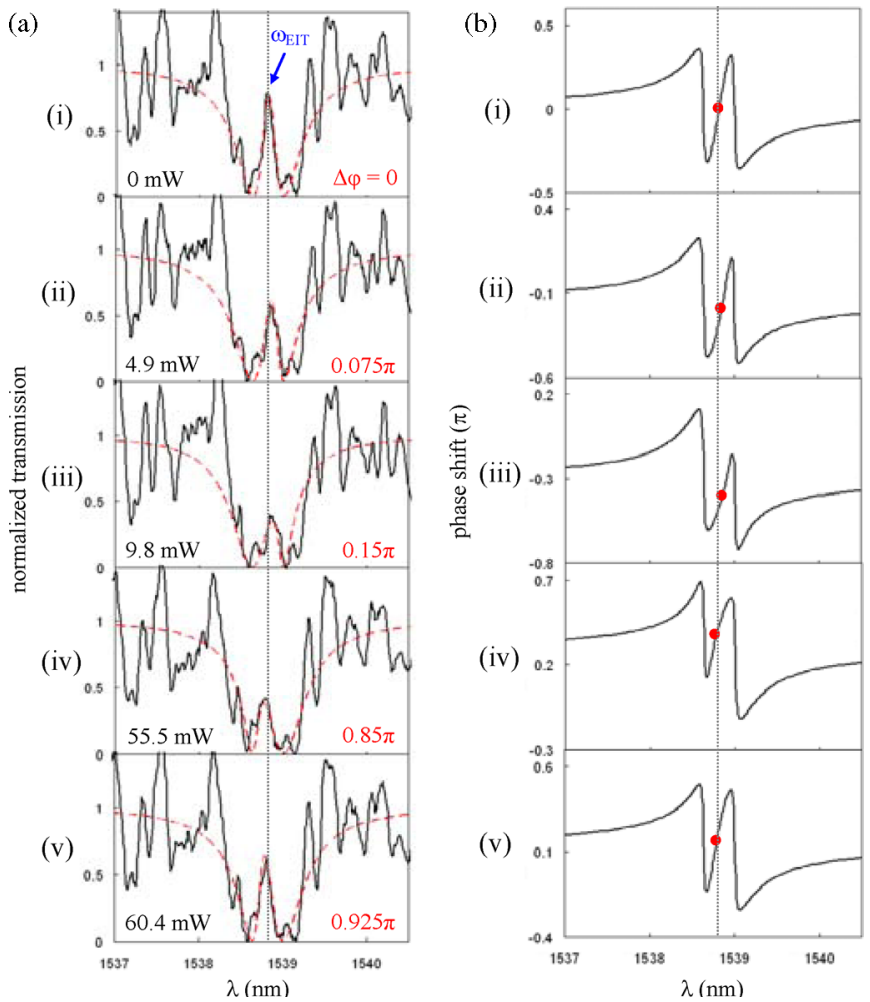

Fig. 7. (a) Measured and theoretical transmission lineshapes with various phase difference $\Delta \phi$ at $\delta=1.92$ for sample $4(L=142 a)$. Solid black lines show experimental data and red dashed lines show theoretical fits. (b) Corresponding theoretical transmission phase shift. The dotted gray lines show the position of the EIT-like transparence peak for $\Delta \phi=0$.

two pump beams are aligned with one at cavity 1 to perturb the resonance and the other at the connecting photonic crystal waveguide to tune the phase difference $\phi$. At first, the pump power of the first laser is adjusted to decrease the wavelength detuning $\delta$ from 16.69 to 1.92 and reach the optical EIT-like regime $\left(\delta<\sim 3.5\right.$ ), where the tuned $\lambda_{1}=1538.64 \mathrm{~nm}$ and $\lambda_{2}$ does not change. Then, the pump power of the second laser is tuned to control the phase difference $\phi$. When the round-trip phase $2 \phi$ is close to $2 n \pi$, the transmission spectrum exhibits a narrow and symmetric EIT-like peak, as shown in Fig. 7(a)(i), where the FWHM of the transparency peak is $\sim 0.16 \mathrm{~nm}$, corresponding to a $Q_{\text {EIT }}$ of 9600 . The phase shift $\Delta \phi$ per milliwatt pump power is linear and $\sim 0.0153 \pi / \mathrm{mW}$. As the pump power of the second laser is increased, $\Delta \phi$ is deterministically controlled between the full range from 0 to $\pi$. For example, Fig. 7(a)(ii) illustrates a phase control from 0 to $0.075 \pi$ and Fig. 7(a)(iii) illustrates a control from 0 to $0.15 \pi$. The line shapes are less symmetric and are tilted to the longer wavelengths, and the center peaks are broader with a lower transmission. Both cavity resonances are not shifted, which indicates independent $\delta$ and $\phi$ tuning have now been achieved. When $\Delta \phi=0.5 \pi$, the roundtrip phase is $\pi$, and indeed, no EIT-like interference is observed, further verifying our experiments. As $\Delta \phi$ is increased continuously above $0.5 \pi$, the transparency peaks appear again and are titled to the shorter wavelengths, as shown in Fig. 7(a)(iv) and (v). (a)

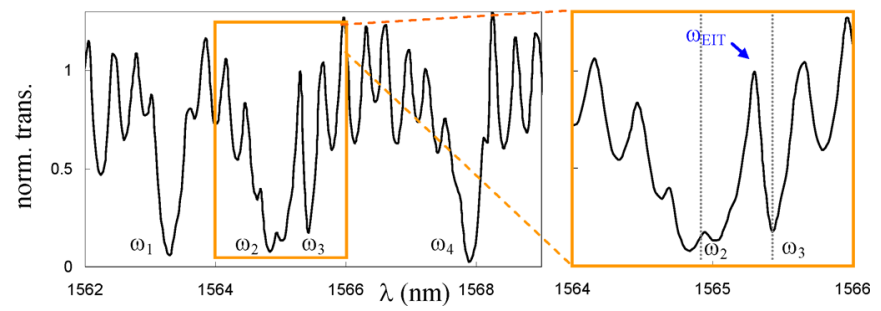

Fig. 8. (a) Measured transmission lineshapes on six-cavity resonance system (sample 5). Resonances 2 and 3 are sufficiently close to observe interference. (b) Expanded spectral measurements for resonances 2 and 3 depicted the interference peak.

Fig. 8 illustrates measurements of a six-cavity resonance system where two resonances are brought into spectral overlap $\left(\omega_{2}\right.$ and $\left.\omega_{3}\right)$. In this example, the loaded cavity $Q$ is $\sim 6000$ and, as in the earlier measurements, the transmission contrast for each cavity is $\sim 15 \mathrm{~dB}$. Fig. 8(b) shows an expanded detail of the cavity interferences, with the transparency peak at $\sim 1565.3 \mathrm{~nm}$.

\section{TheORETICAL ANALYSIS}

The measured transmission spectra are examined with the coupled-mode formalism [37], with the theoretical model shown in Fig. 2(a). The EIT-like system consists of a waveguide sidecoupled to two cavities, where $a$ is the amplitude of the cavity mode that is normalized to represent the energy of the cavity mode $U=|a|^{2}$, and $s$ is the amplitude of the waveguide mode that is normalized to represent the power of the waveguide mode $P=|s|^{2}$. The two side-coupled cavity mode amplitudes are represented by [38], [39]

$\frac{d a_{1}}{d t}=\left(-\frac{1}{2 \tau_{\text {total }, 1}}+i\left(\omega_{1}+\Delta \omega_{1}-\omega_{\mathrm{wg}}\right)\right) a_{1}+\kappa s_{1+}+\kappa s_{L}$

$\frac{d a_{2}}{d t}=\left(-\frac{1}{2 \tau_{\text {total }, 2}}+i\left(\omega_{2}+\Delta \omega_{2}-\omega_{\mathrm{wg}}\right)\right) a_{2}+\kappa s_{R}+\kappa s_{2+}$.

As shown in Fig. 2(a), $s_{1-}=\exp (-i \phi) s_{L}+\kappa a_{1}, s_{R}=$ $\exp (-i \phi) s_{1+}+\kappa a_{1}, s_{L}=\exp (-i \phi) s_{2+}+\kappa a_{2}$, and $s_{2-}=$ $\exp (-i \phi) s_{R}+\kappa a_{2}$, with the transmission coefficient $T=$ $\left|s_{2-}\right|^{2} /\left|s_{1+}\right|^{2}$ solved numerically. $\kappa$ is the coupling coefficient between the waveguide mode $s(t)$ and the cavity resonance mode $a(t)$, and is expressed by $\kappa=i \exp (-i \phi / 2) / \sqrt{2 \tau_{c}}$. $\phi=\omega_{\mathrm{wg}} n_{\mathrm{eff}} L / c$ is the phase difference between the two cavities, where $n_{\mathrm{eff}}$ is the effective index of fundamental mode $(2.768$ at $1.55 \mu \mathrm{m})$. The total loss rate for the resonance mode $1 / \tau_{\text {total }}$ [32] is $1 / \tau_{\text {total }}=1 / \tau_{c}+1 / \tau_{\text {int }}$, where $1 / \tau_{c}\left(=\omega / Q_{c}\right)$ and $1 / \tau_{\text {int }}\left(=\omega / Q_{\text {int }}\right)$ are the decay rates from the cavity into the waveguide and into the continuum, respectively.

The calculated transmissions are then shown in the dashed curves of Figs. 6 and 7. The theoretical results show remarkable fit with the experimental transmissions. The cavity resonance is obtained from the minima of the transmission spectra. $Q_{\text {int }}$ is 
$\sim 60,000$ from the measurements and 3-D FDTD simulations. $Q_{c}$ of each cavity is slightly adjusted around the measured values to fit the background shape of the transmission dip. The phase difference $\phi$ is tuned to obtain the similar transmission lineshapes as experimental data. When the round-trip phase $2 \phi$ is close to $2 n \pi$, the transmission spectrum exhibits a narrow and almost symmetric EIT-like peak, as shown in Fig. 6(a)(ii)-(iv) and(b)(ii)-(v) and Fig. 7(a). For samples 2 and 3, as the phase difference shifts away from $2 n \pi$, the asymmetric Fano-like lineshape is observed at one side of the transmission dip due to a small wavelength detuning $\delta$, as shown in Fig. 6(a)(v) and (vi) and (b)(vi). For sample 4, as the phase difference $\phi$ is shifted away from 0 or $\pi$, the EIT-like peaks are less symmetric and get broader, as shown in Fig. 7(a). Fig. 7(b) show the calculated corresponding transmission phase shift for the respective samples. In the EIT-like transparency region, the phase slope is positive and shows a steep linear normal dispersion. As the coupling strength between two cavities is increased and tuned by the pump beam, the slope is steeper, indicating a longer photon delay. The red dots show the locations of EIT peaks or Fano-like transitions where slow light is expected, in addition to the fast light regions at two cavity resonances.

\section{CONCLUSION}

In summary, we demonstrate experimentally the deterministic tuning of all-optical analog to electromagnetically induced transparency in coherently coupled silicon photonic crystal cavities, with both resonance- and phase tuning control. In our cavities with wavelength-scale localization, distinctive EIT- and Fano-like lineshapes are observed in the resonance- and phasematched coupled cavity coherent interactions, with measured EIT-like linewidths narrower than individual resonances. The maximum $Q$ of our measured transparency resonance is 13,000 , corresponding to an optical delay up to $20 \mathrm{ps}$. Our experimental and theoretical results support efforts toward realization of photon pulse trapping, dynamic bandwidth compression, and all-optical dynamical storage of light in scalable photonic crystal cavity arrays.

\section{ACKNOWLEDGMENT}

The authors acknowledge discussions with S. Kocaman.

\section{REFERENCES}

[1] T. Tanabe, M. Notomi, E. Kuramochi, A. Shinya, and H. Taniyama, "Trapping and delaying photons for one nanosecond in an ultra-small high- $Q$ photonic-crystal nanocavity," Nature Photon., vol. 1, pp. 49-52, 2007.

[2] S. Noda, M. Fujita, and T. Asano, "Spontaneous-emission control by photonic crystals and nanocavities," Nature Photon., vol. 1, pp. 449-458, 2007.

[3] T. Tanabe, M. Notomi, S. Mitsugi, A. Shinya, and E. Kuramochi, "Alloptical switches on a silicon chip realized using photonic crystal nanocavities," Appl. Phys. Lett., vol. 87, pp. 151112-1-151112-3, 2005.

[4] X. Yang, C. Husko, M. Yu, D. L. Kwong, and C. W. Wong, "Observation of femtojoule optical bistability involving Fano resonances in high$Q / V_{m}$ silicon photonic crystal nanocavities," Appl. Phys. Lett., vol. 91, pp. 051113-1-051113-3, 2007.

[5] X. Yang and C. W. Wong, "Coupled-mode theory for stimulated Raman scattering in high- $Q / V_{m}$ silicon photonic band gap defect cavity lasers," Opt. Exp., vol. 15, pp. 4763-4780, 2007.
[6] H. Rong, R. Jones, A. Liu, O. Cohen, D. Hak, A. Fang, and M. Paniccia, "A continuous-wave Raman silicon laser," Nature, vol. 433, pp. 725-728, 2005.

[7] X. Yang, C. Chen, C. Husko, and C. W. Wong, "Digital resonance tuning of high- $Q / V_{m}$ silicon photonic crystal nanocavities by atomic layer deposition," Appl. Phys. Lett., vol. 91, pp. 161114-1-161114-3, 2007.

[8] R. Bose, X. Yang, R. Chatterjee, J. Gao, and C. W. Wong, "Weak coupling interactions of colloidal lead sulphide nanocrystals with silicon photonic crystal nanocavities near $1.55 \mu \mathrm{m}$ at room temperature," Appl. Phys. Lett., vol. 90, pp. 111117-1-111117-3, 2007.

[9] Y. Akahane, T. Asano, H. Takano, B.-S. Song, Y. Takana, and S. Noda, "Two-dimensional photonic-crystal-slab channeldrop filter with flat-top response," Opt. Exp., vol. 13, pp. 2512-2530, 2005.

[10] Y. Tanaka, J. Upham, T. Nagashima, T. Sugiya, T. Asano, and S. Noda, "Dynamic control of the $Q$ factor in a photonic crystal nanocavity," Nature Mater, vol. 6, pp. 862-865, 2007.

[11] S. E. Harris, "Electromagnetically induced transparency," Phys. Today, vol. 50, pp. 36-42, 1997.

[12] M. F. Yanik, W. Suh, Z. Wang, and S. Fan, "Stopping light in a waveguide with an all-optical analog of electromagnetically induced transparency," Phys. Rev. Lett., vol. 93, pp. 233903-1-233903-4, 2004.

[13] M. F. Yanik and S. Fan, "Time reversal of light with linear optics and modulators," Phys. Rev. Lett., vol. 93, pp. 173903-1-173903-4, 2004.

[14] S. Fan, M. F. Yanik, M. L. Povinelli, and S. Sandhu, "Dynamic photonic crystals," Opt. Photon. News, vol. 18, pp. 41-45, 2007.

[15] W. Suh, Z. Wang, and S. Fan, "Temporal coupled-mode theory and the presence of non-orthogonal modes in lossless multimode cavities," IEEE J. Quantum Electron., vol. 40, no. 10, pp. 1511-1518, Oct. 2004.

[16] S. Fan, M. F. Yanik, Z. Wang, S. Sandhu, and M. L. Povinelli, "Advances in theory of photonic crystals," J. Lightw. Technol., vol. 24, no. 12, pp. 4493-4501, Dec. 2006.

[17] D. D. Smith, H. Chang, K. A. Fuller, A. T. Rosenberger, and R. W. Boyd, "Coupled-resonator-induced transparency," Phys. Rev. A, Gen. Phys., vol. 69, pp. 063804-1-063804-6, 2004.

[18] L. Maleki, A. B. Matsko, A. A. Savchenkov, and V. S. Ilchenko, "Tunable delay line with interacting whispering-gallery-mode resonators," Opt. Lett., vol. 29, pp. 626-628, 2004.

[19] K. Totsuka, N. Kobayashi, and M. Tomita, "Slow light in coupledresonator-induced transparency," Phys. Rev. Lett., vol. 98, pp. 213904-1213904-4, 2007.

[20] A. Naweed, G. Farca, S. I. Shopova, and A. T. Rosenberger, "Induced transparency and absorption in coupled whispering-gallery microresonators," Phys. Rev. A, Gen. Phys., vol. 71, pp. 043804-1-043804-4, 2005.

[21] S. T. Chu, B. E. Little, W. Pan, T. Kaneko, and Y. Kokebun, "Second-order filter response from parallel coupled glass microring resonators," IEEE Photon. Technol. Lett., vol. 11, no. 11, pp. 1426-1428, Nov. 1999.

[22] Q. Xu, S. Sandhu, M. L. Povinelli, J. Shakya, S. Fan, and M. Lipson, "Experimental realization of an on-chip all-optical analogue to electromagnetically induced transparency," Phys. Rev. Lett., vol. 96, pp. 1239011-123901-4, 2006.

[23] Q. Xu, J. Shakya, and M. Lipson, "Direct measurement of tunable optical delays on chip analogue to electromagnetically induced transparency," Opt. Exp., vol. 14, pp. 6463-6468, 2006.

[24] Q. Xu, P. Dong, and M. Lipson, "Breaking the delay-bandwidth limit in a photonic structure," Nature Phys., vol. 3, pp. 406-410, 2007.

[25] L. V. Hau, S. E. Harris, Z. Dutton, and C. H. Behroozi, "Light speed reduction to 17 metres per second in an ultracold atomic gas," Nature, vol. 397, pp. 594-598, 1999.

[26] J. J. Longdell, E. Fraval, M. J. Sellars, and N. B. Manson, "Stopped light with storage times greater than one second using electromagnetically induced transparency in a solid," Phys. Rev. Lett., vol. 95, pp. 063601-1063601-4, 2005.

[27] X. Yang, M. Yu, D.-L. Kwong, and C. W. Wong, "All-optical analogue to electromagnetically induced transparency in multiple coupled photonic crystal cavities," Phys. Rev. Lett., vol. 102, pp. 173902-1-173902-4, 2009.

[28] J. Pan, Y. Huo, K. Yamanaka, S. Sandhu, L. Scaccabarozzi, R. Timp, M. L. Povinelli, S. Fan, M. M. Fejer, and J. S. Harris, "Aligning microcavity resonances in silicon photonic-crystal slabs using laser-pumped thermal tuning," Appl. Phys. Lett., vol. 92, pp. 103114-1-103114-3, 2008.

[29] Y. Akahane, T. Asano, B. S. Song, and S. Noda, "High- $Q$ photonic nanocavity in a two-dimensional photonic crystal," Nature, vol. 425 , pp. 944-947, 2003.

[30] A. Farjadpour, D. Roundy, A. Rodriguez, M. Ibanescu, P. Bermel, J. D. Joannopoulos, S. G. Johnson, and G. Burr, "Improving accuracy 
by subpixel smoothing in FDTD," Opt. Lett., vol. 31, pp. 2972-2974, 2006.

[31] A. Chutinan, M. Mochizuki, M. Imada, and S. Noda, "Surface-emitting channel drop filters using single defects in two-dimensional photonic crystal slabs," Appl. Phys. Lett., vol. 79, pp. 2690-2692, 2001.

[32] Y. Akahane, T. Asano, B.-S. Song, and S. Noda, "Fine-tuned high- $Q$ photonic-crystal nanocavity," Opt. Exp., vol. 13, pp. 1202-1214, 2005.

[33] A. B. Matsko, A. A. Savchenkov, D. Strekalov, V. S. Ilchenko, and L. Maleki, "Interference effects in lossy resonator chains," J. Mod. Opt., vol. 51, pp. 2515-2522, 2004.

[34] M. Skorobogatiy, G. Bégin, and A. Talneau, "Statistical analysis of geometrical imperfections from the images of 2D photonic crystals," Opt. Exp., vol. 13, p. 2487, 2005.

[35] T. Asano, W. Kunishi, M. Nakamura, B. S. Song, and S. Noda, "Dynamic wavelength tuning of channel-drop device in two-dimensional photonic crystal slab," Electron. Lett., vol. 41, pp. 37-38, 2005.

[36] S. McNab, N. Moll, and Y. Vlasov, "Ultra-low loss photonic integrated circuit with membrane-type photonic crystal waveguides," Opt. Exp., vol. 11, pp. 2927-2939, 2003.

[37] H. A. Haus, "Coupling of modes-Resonators and couplers," in Waves and Fields in Optoelectronics. Englewood Cliffs, NJ: Prentice-Hall 1984, ch. 7, pp. 197-204.

[38] S. Fan, W. Suh, and J. D. Joannopoulos, "Temporal coupled-mode theory for the Fano resonance in optical resonators," J. Opt. Soc. Amer. A, Opt. Image Sci., vol. 20, pp. 569-572, 2003.

[39] B. Maes, P. Bienstman, and R. Baets, "Switching in coupled nonlinear photonic-crystal resonators," J. Opt. Soc. Amer. B, Opt. Phys., vol. 22 pp. 1778-1784, 2005.

Xiaodong Yang (S'01-M'09) received the B.Sc. degree from the University of Science and Technology of China, Hefei, P.R. China, in 2000, the M.Sc. degree from the Chinese Academy of Sciences, Beijing, P.R. China, in 2003, and the Ph.D. degree from Columbia University, New York in 2009.

$\mathrm{He}$ is currently a Postdoctoral Fellow at the University of California at Berkeley and Lawrence Berkeley National Laboratory. Previously he was with the Optical Nanostructures Laboratory, Columbia University. He was supported by an Intel Foundation Ph.D. Fellowship from 2006 to 2008. His current research interests include silicon nanophotonics and photonic crystals, surface plasmons and subwavelength plasmonic devices, and especially the light-matter interactions with photonic crystal cavities and plasmonic nanocavities.

Dr. Yang received the Chinese Government Award for Outstanding Student Abroad in 2008 .

Mingbin Yu received the B.S. degree in physics from Xi' an University of Technology, Xi' an, P.R. China, in 1982, and the M.Eng. and Ph.D. degrees in semiconductor and microelectronics from Xi'an Jiaotong University, Xi'an, in 1989 and 1995, respectively.

He was Professor, Director of the Department of Applied Physics, and Vice Dean of the School of Science, Xi'an University of Technology, before he joined Nanyang Technological University as a Research Fellow in 1998. In 2000, he joined the Institute of Microelectronics, Singapore, where he is a Member of Technical Staff. He is the author or couathor of more than 80 papers published in journals and conferences. His current research interests include silicon electronic-photonic devices and IC technology.
Dim-Lee Kwong (A'84-SM'90-F'09) is currently the Executive Director of the Institute of Microelectronics, Singapore, and a Professor of electrical and computer engineering at the National University of Singapore, Singapore. He was Earl N. and Margaret Bransfield Endowed Professor at the University of Texas at Austin from 1990 to 2007, and the Temasek Professor of the National University of Singapore from 2001 to 2004. He is the author or coauthor of more than 900 referred archival publications (500 journal and 400 conference proceedings) and has presented more than 70 invited talks at international conferences. He is the holder of more than 23 U.S. patents. More than 55 students received their Ph.D. degrees under his supervision. His research interests include CMOS-compatible Si/Ge nanowire devices and circuits for future CMOS technology and applications, silicon electronic-photonic IC technology and applications, Si-based biosensors and integrated lab-on-chip, and sensors, actuators, and integrated microsystems.

Prof. Kwong received the IBM Faculty Award in 1984-1986, the Semiconductor Research Corporation Inventor Award in 1993-1994, the General Motor Foundation Fellowship in 1992-1995, the Halliburton Foundation Excellent Teaching Award in 1994, the Engineering Foundation Award in 1995, and the IEEE George Smith Award in 2007.

Chee Wei Wong (S'03-M'09) received the B.Sc. degree (with highest honors) in mechanical engineering and the B.A. degree (with highest honors) in economics from the University of California at Berkeley, Berkeley, during 1996-1999, and the Masters of Science and Doctorate of Science degrees from Massachusetts Institute of Technology, Cambridge, in 2001 and 2003, respectively.

He was a Postdoctoral Research Associate at the MIT Microphotonics Center in 2003. He is currently with the Optical Nanostructures Laboratory, Columbia University, New York, which he joined in 2004. Since 2003, he has given more than 30 invited talks, and published more than 30 journal articles, more than 45 conference papers, and two book chapters. He is the holder of six awarded patents and 10 pending patents.

Dr. Wong is a member of the American Physical Society, the American Society of Mechanical Engineers, the Optical Society of America, and the Sigma Xi. He was the recipient of the 2009 3M Faculty award, the 2008 National Science Foundation CAREER Award, and the 2007 Defense Advanced Research Projects Agency Young Faculty Award. His current research interests include nonlinear and quantum optics in nanophotonics, silicon electronic-photonic circuits and photonic crystals, quantum dot interactions in nanocavities, nanoelectromechanical systems, and nanofabrication. 